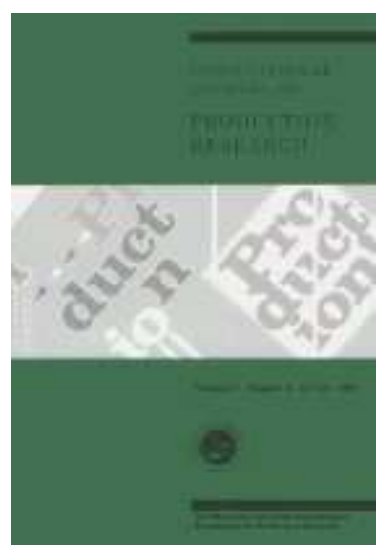

\title{
Optimization of a Set of Xbar or Principal Components Control Charts Using Genetic Algorithms
}

\begin{tabular}{|c|c|}
\hline Journal: & International Journal of Production Research \\
\hline Manuscript ID: & TPRS-2008-IJPR-0840.R1 \\
\hline Manuscript Type: & Original Manuscript \\
\hline $\begin{array}{r}\text { Date Submitted by the } \\
\text { Author: }\end{array}$ & 26-May-2009 \\
\hline Complete List of Authors: & $\begin{array}{l}\text { Aparisi, Francisco; Universidad Politecnica de Valencia, } \\
\text { Departamento de Estadistica e I O } \\
\text { De Luna, Marco } \\
\text { Epprecht, Eugenio }\end{array}$ \\
\hline Keywords: & QUALITY CONTROL, GENETIC ALGORITHMS \\
\hline Keywords (user): & QUALITY CONTROL, GENETIC ALGORITHMS \\
\hline
\end{tabular}

\section{scholarONE" \\ Manuscript Central}




\title{
Optimization of a Set of $\bar{X}$ or Principal Components Control Charts Using Genetic Algorithms
}

\author{
Francisco Aparisi ${ }^{\mathrm{a}}$, Marco A. de Luna ${ }^{\mathrm{b}}$, Eugenio Epprecht ${ }^{\mathrm{c}}$ \\ a Departamento de Estadística e Investigación Operativa Aplicadas y Calidad, Universidad Politécnica \\ de Valencia, 46022 Valencia, Spain. \\ ${ }^{\mathrm{b}}$ Departamento de Ingeniería Industrial y Mecánica, ITESM, Campus Guadalajara, 45140 Guadalajara, \\ Mexico \\ c Departamento de Engenharia Industrial, Pontificia Universidade Católica de Río de Janeiro \\ CEP: 22453-900, Gávea, Rio de Janeiro - RJ - Brasil
}




\title{
Optimization of a Set of $\bar{X}$ or Principal Components Control Charts Using Genetic Algorithms
}

\begin{abstract}
When a multivariate process is to be monitored, there are the options of employing a set of univariate control charts or a single multivariate chart. This paper shows how to effectively design a multivariate control scheme consisting of two or three $\bar{X}$ charts, using Genetic Algorithms to optimize the charts parameters. The procedure is implemented using software tools, which we designed. A complete performance comparison of the scheme with the Hotelling's $\mathrm{T}^{2}$ control chart can be made in order to help the user in choosing the most adequate option for the process under consideration. Also, if the user prefers to employ charts based on principal components rather than on the original variables, the software can be used in the same way to optimize a set of two or three control charts based on these components, and to compare their performance with the performance of the $\mathrm{T}^{2}$ chart on the principal components.
\end{abstract}

Keywords: Control Chart, Optimization, multiple, Genetic Algorithms. 6161 words.

\section{1.- Introduction}

One of the current needs of industry is the ability to control simultaneously $p$ correlated quality variables of a product or productive process. It is well known (Montgomery, 2005) that there are two main alternatives for this purpose: the first one is to use $p$ univariate control charts simultaneously, one for each variable (normally a set of $\bar{X}$, EWMA or CUSUM charts). The second option is using a single multivariate chart. Among the available choices of multivariate charts for controlling a vector of $p$ means are Hotelling's $T^{2}$ chart (Hotelling, 1947; Champ and Aparisi, 2008), MEWMA (Lowry et al., 1992) and MCUSUM charts (Crosier, 1988; Pignatiello and Runger, 1990). Lowry and Montgomery (1995) present a review of 
the literature on control charts for multivariate quality control, describing these procedures, among others.

The essential idea behind the use of a multivariate control chart is to take into account the correlation structure of the variables, which expectedly should lead to greater sensitivity of the control chart to changes in the mean vector. Nevertheless, the performance comparison between a set of univariate charts and one multivariate control chart is complicated. None of the alternatives is uniformly better than the other, and the best alternative will depend on the number of variables, on their correlation structure and on the direction of the mean shift (see Runger and Montgomery, 1997; Lowry and Montgomery, 1995). For example, in the case of two variables, when the shift occurs along the first principal component axis (the principal axis of the elliptical in-control region), the set of $\bar{X}$ control charts is faster in signalling than the $\mathrm{T}^{2}$ control chart. However, for other shift directions, like along the second principal component, the $\mathrm{T}^{2}$ control chart shows clearly better performance.

Another problem for the practitioner in the case of multiple $\bar{X}$ charts is how to set the values for the control limits in order to guarantee the desired protection against false alarms. Note that the Bonferroni and Dunn-Sidak corrections (Hayter and Tsui, 1994; Johnson and Wichern, 2007; Dunn, 1958; Sidak, 1967), conceived for adjusting the individual type I risks of a set of univariate tests of hypotheses in order to keep the overall risk at a specified low level, assume that the variables are independent. Hence, for the typical case of correlated variables, these corrections are conservative and result in charts that are not very powerful, as stressed by Doganaksoy et al. (1991), Hayter and Tsui (1994) and Serel et al. (2000). To overcome this limitation and take into account the correlations between the variables, Hayter and Tsui (op. cit.) propose a control procedure based on simultaneous exact confidence intervals for the means of the variables, where the critical points are determined using the multivariate normal distribution.

This problem of choosing the control limits for simultaneous univariate charts becomes even more complex when the rapid detection of shifts in some directions is more important than in other directions, or when the magnitudes (in number of standard deviations) of the shifts that are considered relevant are different for each 
variable, because in these cases the best choice of control limits are limits that correspond to unequal type I risks for the individual variables while still maintaining the overall false-alarm rate at the desired level, as already pointed out by Serel et al. (2000).

The use of a single multivariate chart, on the other hand, has the limitation that when the chart signals there is no indication of which process variable was responsible for the signal, and additional procedures should be employed for such identification. For such purpose, Alt (1984) and Doganaksoy et al. (1991) describe a method based on the individual $p$-values for each of the variables. Mason, Tracy and Young (1997) and Mason and Young (1999) propose a method based on the decomposition of the $\mathrm{T}^{2}$ statistic. However, the use of a neural network (Aparisi, Avendaño and Sanz, 2006), outperforms this decomposition in many cases. Other techniques to interpret the signal of a $\mathrm{T}^{2}$ control chart can be found in Runger and Montgomery (1996) and in Kourti and MacGregor (1996), to mention just some of the numerous methods proposed in the literature.

With a separate chart for each variable, in contrast, when one of the charts signals, this indicates directly the variable whose mean has probably shifted. Such an advantage, however, may disappear in the case of simultaneous shifts in several variables, where it seems unlikely that the $\bar{X}$ charts of the variables that have shifted will all signal at the same sample. The method of simultaneous confidence intervals for the individual variables proposed by Hayter and Tsui (1994) has the same diagnostic feature, and may be considered as equivalent to multiple univariate charts, with the improvement, already mentioned, that the critical points are established taking into account the correlations between the variables and result, therefore, in the exact type I risk that has been specified and, correspondingly, in a more powerful control procedure.

As a general conclusion of all that has been said, there is no simple rule for guiding the practitioner who needs to monitor a specific multivariate process in order to determine which control scheme to use. The best performing scheme will depend on a number of process and shift characteristics; hence, to make the best choice the user 
needs to evaluate the performance of each scheme (multiple $\bar{X}$ and $T^{2}$ ) for his/her particular case. Also, he or she may have the problem of specifying the control limits for the charts, taking into account that the schemes performance will depend upon these limits.

An additional degree of complexity in the decision-making process arises given that a number of authors recommend working with the principal components (PCs) of the original variables rather than working with the original variables themselves (Jackson, 1985, 1991; Schall \& Chandra, 1987; Runger \& Alt, 1996; Johnson \& Wichern, 2007; Runger \& Montgomery, 1997; Johnson \& Wichern, 2007). The alternatives in this case are the same as in the case of working with the original variables: either using simultaneous "univariate" charts on some or all of the principal components, or using a multivariate chart on these components. Working with just a few PCs rather than with the original variables has the advantage of reducing the dimensionality of the problem (and, in the case of multiple charts, of reducing the number of charts to maintain), but one must be careful with other aspects such as loss of information or sensitivity to some directions of shift. The issues of how to select which principal components to retain are out of the scope of this paper, and are discussed in Runger \& Alt (1996). See also Johnson \& Wichern (2007).

If the user decides to use principal components instead of the original variables, he or she is still left which the choice between using one chart for each PC or using a single multivariate chart, and with the problem of how to set the proper control limits for the $\operatorname{chart}(\mathrm{s})$.

For supporting the user in the joint decision about which control scheme to use for controlling a specific multivariate process, and which parameters to use with the scheme chosen, this paper proposes a procedure for finding the best parameters of a set of $\bar{X}$ control charts ( 2 and 3 variables), given the desired overall in-control ARL and the shift against which maximum protection is desired. The parameters found are the control limits and sample sizes which minimize the out-of-control ARL for the specified shift. Like in Hayter and Tsui (1994) and in Serel et al. (2000), exact ARL values are calculated through integration of the multivariate normal distribution. In 
addition, like Serel et al. (op. cit.), we do not impose the restriction that the individual type I risks must be identical for different charts in order to optimize the power of the joint scheme for the specified shifts. Unlike them, however, who use an economic model, our approach is to minimize the out-of-control ARL, requiring less information from the practitioner.

The procedure is implemented in a computer program which, in addition to the optimum sample size and control limits for the $\bar{X}$ charts, provides plots of the ARL values (ARL versus the shifts in the variables) for each control scheme (the multiple $\bar{X}$ charts and the equivalent $\mathrm{T}^{2}$ chart with the same in-control ARL and the same sample size). The ARL comparison enables the user to decide which control scheme to employ for each real case, while providing the optimal design parameters for the scheme. This optimization is carried out using Genetic Algorithms.

The user who decides to employ charts based on principal components can also use the program to find the optimal limits for a set of individual $\bar{X}$ charts on the PCs. It is sufficient to enter the problem specifications in an appropriate form into the interface window. Namely, the correlations should all be set to zero (since the principal components are orthogonal), and the relevant shift in the original variables should be converted to its equivalent in terms of the principal components. This conversion is straightforward, as shown in Section 5.

The remainder of this paper is structured as follows: Section 2 describes Shewhart's $\bar{X}$ chart and presents the performance measures and design parameters for a set of $\bar{X}$ charts for monitoring a multivariate process; Section 3 formalizes the problem of designing this set of $\bar{X}$ charts as an optimization problem; Section 4 describes the method and software developed for solving this problem, as well as the software's user interface, and illustrates the use of the software with a number of examples; Section 5 shows how the program can be used to design a set of $\bar{X}$-like charts on the principal component variables rather than on the original variables; and Section 6 summarizes the conclusions of the work. 


\section{2.- Multiple $\bar{X}$ control charts: construction and performance}

\section{measure}

Shewhart's $\bar{X}$ control chart consists of plotting the values of the statistic $\bar{X}$ of successive samples on a chart with upper control limit $U C L=\mu_{0}+k_{U} \frac{\sigma_{0}}{\sqrt{n}}$, and lower control limit $L C L=\mu_{0}-k_{L} \frac{\sigma_{0}}{\sqrt{n}}$, where $\mu_{0}$ and $\sigma_{0}$ are the in-control mean and standard deviation of the process, respectively, and $k_{U}$ and $k_{L}$ are design parameters. The standard practice in univariate process control is to set $k_{U}=k_{L}=3$, what is called "3sigma limits", although one may also use the value for $k_{U}=k_{L}$ that corresponds to a specified false-alarm risk - "probability limits" (Montgomery, 2005). In the case of simultaneous univariate charts for multivariate process control, it is usual to use a common value of $k_{U}=k_{L}$ for all charts, corresponding to an overall false-alarm risk. The approach in this paper, however, is to find the values of $k_{U}$ and $k_{L}$ for each chart that optimize the performance of the set of charts.

One of the most widely used performance measures of a control chart is the Average Run Length (ARL), which is the expected number of points plotted in the chart until an out-of-control signal occurs. For the $\bar{X}$ chart, it is easy to verify, and also well known, that $A R L=[1-\mathrm{P}(\mathrm{LCL}<\overline{\mathrm{X}}<\mathrm{UCL})]^{-1}$, where the value in square brackets is the probability that the sample point falls outside the control limits. This probability corresponds therefore to the false-alarm risk in the case where the process is in control (with mean $\mu_{0}$ ), and to the probability of a true alarm when the process is out of control, with mean $\mu_{1} \neq \mu_{0}$ (the case of changes in the process variance is not within the scope of this paper, so it will be assumed that the process standard deviation is always equal to $\sigma_{0}$ ). When the process is in control (no shifts in the mean) it is easy to check that for a Shewhart's chart $A R L=1 / \alpha$, where $\alpha$ is the probability that the value of $\bar{X}$ is plotted outside control limits, i. e., $\alpha$ is the false-alarm probability for each sample. When the process is out of control (there is a shift in the mean), ARL $=1 /$ (1$\beta$ ), where $\beta$ is the probability that the value of $\bar{X}$ falls between the control limits, i. e., $\beta$ is the probability of the chart does not show an out-of-control signal. 
A user wanting to control $p$ means with $p \bar{X}$ charts must decide the values for the following parameters:

Upper and Lower control limits for each of the $p$ charts: $k_{L_{1}}, k_{U_{1}}, k_{L_{2}}, k_{U_{2}}, \cdots, k_{L_{p}}, k_{U_{p}}$

Sample size: $n$, the same for all the charts, or possibly different sample sizes for each chart. $n_{1}, n_{2}, \ldots, n_{p}$.

This last option of possibly different sample sizes, although currently unusual, can in some cases be optimal and allow a reduction in sampling costs. In the equal-samplesize version of the problem, there are $2 p+1$ parameters to be found; in the possiblydifferent-sample-sizes version, $3 p$ parameters. This last option, possible different sample sizes, although apparently not very common in real applications, may be optimal in some cases and may permit the reduction of sampling costs when they are high. In the equal-sample-size version of the problem, there are $2 p+1$ parameters have to be found; in the possibly-different-sample-sizes version, there are $3 p$ parameters.

The shift size for each variable $\left(X_{i}\right), i=1,2, \ldots, p$, is measured in standard deviation units:

$$
d_{i}=\frac{\mu_{1, i}-\mu_{0, i}}{\sigma_{i}}
$$

where $\mu_{0, i}$ and $\mu_{1, i}$ are in in-control and out-of-control means of $X_{i}$, respectively, and $\sigma_{i}$ is its standard deviation. Let $\mathbf{d}=\left(d_{1}, d_{2}, \cdots, d_{p}\right)$ represent a shift in the $p$ variables. The in-control ARL of the set of $\bar{X}$ charts is computed as:

$$
\begin{aligned}
& A R L_{\mathbf{d}=\mathbf{0}}=\frac{1}{1-P\left(L C L_{1}<\bar{X}_{1}<U C L_{1} \cap L C L_{2}<\bar{X}_{2}<U C L_{2} \cap \cdots \cap L C L_{p}<\bar{X}_{p}<U C L_{p}\right)}= \\
& =\frac{1}{1-\int_{-k_{L_{1}}}^{k_{U_{1}}} \int_{-k_{L_{2}}}^{k_{U_{2}}} \cdots \int_{-k_{L_{p}}}^{k_{U_{p}}} f\left(z_{1}, z_{2}, \cdots, z_{p}\right) d z_{1} d z_{2} \cdots d z_{p}}
\end{aligned}
$$


where $f\left(z_{1}, z_{2}, \cdots, z_{p}\right)$ is the joint density function of a $p$-variate normal distribution.

For an out-of-control process, $\mathbf{d}=\left(d_{1}, d_{2}, \cdots, d_{p}\right)^{\prime} \neq \mathbf{0}$,

$$
\begin{aligned}
& A R L_{\mathbf{d} \neq \mathbf{0}}=\frac{1}{1-P\left(L C L_{1}<\bar{X}_{1}<U C L_{1} \cap L C L_{2}<\bar{X}_{2}<U C L_{2} \cap \cdots \cap L C L_{p}<\bar{X}_{p}<U C L_{p}\right)}= \\
& =\frac{1}{1-\int_{-k_{L_{1}}-d_{1} \sqrt{n_{1}}}^{k_{U_{1}}-d_{1} \sqrt{n_{1}}} \int_{-k_{L_{2}}-d_{2} \sqrt{n_{2}}}^{k_{U_{2}}-d_{2} \sqrt{n_{2}}} \cdots \int_{-k_{L_{p}}-d_{p} \sqrt{n_{p}}}^{k_{U_{p}}-d_{p} \sqrt{n_{p}}} f\left(z_{1}, z_{2}, \cdots, z_{p}\right) d z_{1} d z_{2} \cdots d z_{p}}
\end{aligned}
$$

Naturally, the user desires an as large as possible in-control ARL, which means few false alarms, and at the same time small ARL values for shifts whose fast detection is considered important. These objectives pose a problem in the design of control charts, which may be formulated as an optimization problem.

\section{3.- Formal definition of the optimization problem}

The design of the multiple $\bar{X}$ charts can be formulated as an optimization problem, in the following way. The user specifies: the overall in-control ARL desired for the set of $p \bar{X}$ control charts, and the shift in the vector of means against which maximum protection (minimum ARL) is required. As for the sample size, the user may choose between using the same sample size for all of the charts, or allow the optimization routine find the best sample size for each chart, in which case the sample sizes may differ for the different variables. In either case, the user should indicate a maximum value for the sample size(s). Formally:

Given:

In-control ARL, $A R L_{\mathbf{d}=\mathbf{0}}$

Shift to be detected with minimum ARL, $\mathbf{d}^{*}=\left(d_{1}, d_{2}, \cdots, d_{p}\right)^{\prime}$

Maximum sample size to be used, $n_{\max }$

Type of problem (equal or possibly different sample sizes)

Find:

Upper and lower control limits for each chart, $k_{L 1}, k_{U 1}, k_{L 2}, k_{U 2}, \ldots, k_{L p}, k_{U p}$ Sample size: common $n$ or $n_{1}, n_{2}, \ldots, n_{p}$. 
Such that:

$$
\begin{aligned}
& \operatorname{ARL}(\mathbf{d}=\mathbf{0})=A R L_{\mathbf{d}=\mathbf{0}} \\
& \operatorname{Min} A R L\left(\mathbf{d}=\mathbf{d}^{*}\right)
\end{aligned}
$$

This optimization problem is not trivial. The mix of continuous and integer variables together with difficult constraints prevent the use of standard optimization routines. In order to solve this, Genetic Algorithms have been employed and user-friendly software has been designed to make the method accessible to any user. GA have been successfully employed previously in the optimization of the parameters of control charts; see, for example, $\mathrm{He}$ and Grigoryan (2006), Aparisi and García-Díaz (2007), Champ and Aparisi (2008) and Aparisi and De Luna (2008). The next section shows the details of the GA and the software developed.

\section{4.- Software and Example of an Application}

We have developed software that gives the solution for the cases $p=2$ and $p=3$ variables. For more than 3 variables, the processing time becomes extremely high due to the necessity of evaluating $p$-dimensional integrals. Besides, one of the objectives of the software is to provide performance comparisons between the multiple $\bar{X}$ charts and the $\mathrm{T}^{2}$ control chart, in order to give support for the user's final decision. Such comparisons are made employing graphics, which are not possible to sketch if $p>3$. With more than 3 variables, however, the user may consider working with just 2 or 3 of the principal components, and the program can be used in this case in optimizing the charts on these components, as will be shown in Section 5.

The program runs on Windows ${ }^{\circledR}$ platform and can be made available from the authors. The software has been written employing CodeGear Delphi ${ }^{\circledR}$, formerly known as Borland Delphi, a software development environment for Microsoft Windows applications. It supports development of native Windows applications in the Delphi programming language, a further development of Object Pascal. The conceptual framework of the software is to provide a friendly interface for the use of Genetic Algorithms to find the best parameters of a set of multiple $\bar{X}$ charts. Similar 
framework has been employed for the optimization of other quality control charts [Aparisi and García-Díaz (2004, 2007) and Champ and Aparisi (2008)].

\section{[INSERT FIGURE 1 OVER HERE]}

As shown, the user has to input the data for the problem in the sub-window "Model Parameters". After the optimization is carried out, the output is shown in the subwindow "Results", where the value for control limits are in sigma units.

Genetic Algorithms (GA) require a fitness function to evaluate the appropriateness of each of the solutions of the population. The quality of this fitness function is fundamental in order for these algorithms to work correctly. The fitness function employed by Aparisi and García-Díaz (2004) for optimizing EWMA and MEWMA charts has been used in this paper, because it adequately solves the problem posed here.

The next equation shows the fitness function employed.

Fitness $=\left\{\begin{array}{l}\max -w_{1}\left|A R L_{d=0}-A R L 0\right|-w_{2} A R L_{d^{*}} \quad \text { if } \quad\left(A R L_{d=0}-A R L 0\right) \leq 0 \\ \max -2 w_{1}\left|A R L_{d=0}-A R L 0\right|-w_{2} A R L_{d^{*}} \quad \text { if } \quad\left(A R L_{d=0}-A R L 0\right)>0\end{array}\right.$

where $A R L_{\mathbf{d}=\mathbf{0}}$ represents the desired in-control ARL, ARL0 represents the ARL for $\mathbf{d}=\mathbf{0}$, calculated for each solution, $A R L_{\mathbf{d}^{*}}$ represents the $A R L$ for $\mathbf{d}=\mathbf{d}^{*}$ (the shift for which we want to minimize the ARL), and $w_{1}$ and $w_{2}$ represent two weights used to achieve a proper balance between the the two parts of the equation. In our case, the weights employed were $w_{1}=1$ and $w_{2}=10$. Weight $w_{1}$ penalizes solutions that have an in-control ARL not close to the desired value, and weight $w_{2}$ penalizes solutions with high out-of-control ARL. The value of "max", the maximum possible fitness value, is not critical, as we are using a binary tournament for the selection procedure. A value of 10000 is employed in the software.

The performance of the GA depends on various parameters: crossover probability, crossover type, among others. An experiment has been carried out to obtain the best parameters for solving this specific problem. For the case $p=2,36$ treatments were 
carried out and 24 treatments were considered for three variables $(p=3)$. The stopping criterion was the number of evaluations. The factor levels considered for $p=$ 2 were: population size $(100,200,400)$, crossover probability $(0.60,0.90)$, mutation probability $(0.03,0.05,0.10)$ and tournament size $(2,4)$. For the case $p=2$ the levels analyzed were: population size $(200,400,500)$, crossover probability $(0.60,0.90)$ and mutation probability $(0.03,0.05,0.10,0.15)$.

The results showed that none of the ANOVA assumptions were satisfied: normality and independence of residuals and homocedasticity. Therefore, it was necessary to increase the number of replications and to analyze the results using a classification tree employing the exhaustive CHAID method. The analysis shows that the significant factors (95\% confidence level) for $p=2$ were: population size, mutation probability and tournament size. The crossover probability has no influence on the fitness value. For the case $p=3$, all the factors were significant. Once the optimum levels have been found, another experiment was carried out to find the best level for the number of evaluations. Table 1 shows the optimum levels for $p=2$ and $p=3$.

\section{[INSERT TABLE 1 OVER HERE]}

The GA routine used includes some of the newest developments in the field, with the aim of increasing the quality of the solutions, Vavak and Fogarty (1996). It consists of a hybrid between the classic GAs, generational type, and the newest steady-state type. Concretely, genetic operators of crossover and mutation are applied to a number $\mathrm{X}$ of individuals selected by binary tournament in the population. We have found that the best performance of the GA is obtained with $\mathrm{X}$ equal to $25 \%$ of the population. After the crossover, the newly generated individuals substitute the $\mathrm{X}$ individuals with lowest fitness in the population. Therefore, only new solutions with better fitness are introduced in the population. The unfeasible solutions (chromosomes) are allowed to stay in the population, although their fitness is quite low (using a fitness penalty). Once the GA stops, a local search around the proposed solution is carried out. In this way, the team "GA + local search" produces a very robust search routine.

Consider the following two application examples. The first case deals with two variables $(p=2)$. It is required that the overall false alarm rate of a set of two $\bar{X}$ 
control charts produce an in-control ARL equal to 370. The ARL for the shift $\mathbf{d}^{*}=$ $(0.7,0.5)^{\prime}$ must be minimized. The maximum sample size to use is $n=5$, and equal sample sizes are to be used for both charts. The correlation between the variables are $r$ $=0.8$. Figure 1 shows the result after the GA ends the optimization, taking only about 8 seconds to finish. The sample size to be used is $n=5$. The value of $k_{L}$ and $k_{U}$ for the control limits of the charts are: $\mathrm{k}_{\mathrm{L} 1}=2.79, \mathrm{k}_{\mathrm{U} 1}=4.25, \mathrm{k}_{\mathrm{U} 2}=3.78, \mathrm{k}_{\mathrm{L} 2}=3.94$. With these parameters the chart detects the shift $\mathbf{d}^{*}=(0.7,0.5)^{\prime}$ with an ARL $=9.05$. As shown by the program, the equivalent $\mathrm{T}^{2}$ control chart has, of course, the same incontrol ARL $=370$, but it is slower in detecting this shift: the out-of-control ARL is 19.90 .

Figure 2 shows the tab "Evaluation vs $\mathrm{T}^{2}(p=2)$ " in the interface window, where a pair of plots compares the performances of the two schemes. The left plot shows in different colours the three regions in the $d_{1} d_{2}$ plane where the ARL of the set of $\bar{X}$ charts has a lower ARL in comparison versus the $\mathrm{T}^{2}$ chart, where the $\mathrm{T}^{2}$ chart is faster in detecting the shift, and where the difference between the two alternatives is not large, i.e. less than $10 \%$. The results of this comparison show that the set of $\bar{X}$ charts is faster in detecting the given shift $\mathbf{d}^{*}$ (point $\mathrm{A}$ on the plot) than the $\mathrm{T}^{2}$ control chart, and is also faster in the area close to the specified shift. This area has a shape according to the correlation between the variables. The plot on the right exhibits the ARL profiles of the two schemes, which is a vertical section of their ARL surfaces: the user should indicate in a field above this plot sub-window (see Figure 2) a fixed value for the shift in one of the variables (in this example, $d_{1}=0.7$ ); the curves plotted by the software tool indicate how each scheme's ARL vary as a function of the shift in the other variable $\left(d_{2}\right.$, in the example shown).

For very large shifts both schemes are very similar, a logical result, as such shifts are easy for any scheme to detect. For a shift that it is not close to the specified shift, $\mathbf{d}^{*}$, the $\mathrm{T}^{2}$ control chart is going to show lower ARLs in comparison with the use of the optimized set of $\bar{X}$ charts. This could be a problem if the possible shifts in the process may occur in whatever location in the $d_{1} d_{2}$ plane. However, this could not be critical if the majority of times where the process shifts will be located close to the specified shift $\mathbf{d}^{*}$. As was commented in the Introduction, the results of the comparison depend on many factors, and the way the process shifts is another factor to consider. This 
software tool helps to understand the differences in performance between the $T^{2}$ and the multiple $\bar{X}$ charts for each specific process under evaluation.

The control limits derived by the software tool are optimal for detecting the given shift and are not necessarily symmetrical. As a consequence, the ARLs of the multiple $\bar{X}$ charts for the symmetric shifts $\mathbf{d}^{*}$ and $-\mathbf{d}^{*}$ are different, and the $\mathrm{T}^{2}$ chart is faster in detecting shifts in the quadrant opposite to the one that contains $\mathbf{d}^{*}$. Again, this may be undesirable or not. It depends on the most common type of shifts in the process. To account for the case in which symmetric shifts $\mathbf{d}^{*}$ and $-\mathbf{d}^{*}$ are equally important, we have designed another program, which finds the optimal parameters for the set of $\bar{X}$ charts, forcing the control limits to be symmetric, i.e., under the constraint of symmetric control limits. The program and its interface are practically identical to those for the asymmetrical case. Figure 3 shows the output for the same case data of the previous example. The ARL for the shift $\mathbf{d}=\mathbf{d}^{*}$ is 13.21 , higher than the ARL with asymmetric limits, but still lower than with the $\mathrm{T}^{2}$ chart. However, the plot for comparison of ARLs shows the symmetry in the performance of the multiple $\bar{X}$ charts in the $d_{1} d_{2}$ plane. Hence, these charts have lost some efficiency in detecting the shift $\mathbf{d}^{*}$ but the shift region where they outperform the $\mathrm{T}^{2}$ control chart becomes larger. The user, knowing the historical behaviour of the process, can choose between using symmetric control limits or not.

\section{[INSERT FIGURE 2 OVER HERE] [INSERT FIGURE 3 OVER HERE]}

In addition, there is an option for obtaining more plots for comparison purposes. Figure 4 shows the plots obtained by clicking on the button "Plot (3d) Solution" in the tab "Optimization". There is in the left a 3D plot (which can be rotated) of the ARL surface for the optimized set of $\bar{X}$ charts and, on the right, the contour plots of the ARL surfaces of the two schemes, superimposed for comparison. It is noticeable that the maximum ARL value for the set of $\bar{X}$ charts is not centred on $(0,0)$ '. However, if the solution is forced to have symmetric control limits the maximum ARL value is centred on the in-control mean of the process (see Figure 5). 


\section{[INSERT FIGURE 4 OVER HERE] [INSERT FIGURE 5 OVER HERE]}

Another example of application follows, for the case $p=3$. The in-control ARL specified for the set of the three $\bar{X}$ control chart is 370 . The shift to minimize the ARL is $\mathbf{d}^{*}=(0.6,0.5,-0.2)^{\prime}$ and the correlations among the variables are: $\mathrm{r}_{12}=0.5, \mathrm{r}_{13}$ $=0.2, \mathrm{r}_{23}=0.5$. The maximum sample size to be used is $n=5$ and the control limits are not forced to be symmetric. Figure 6 shows the results returned by the software, after 75 minutes running on a high performance computer. The control limits are: $\mathrm{k}_{\mathrm{L} 1}$ $=2.79, \mathrm{k}_{\mathrm{U} 1}=4.50, \mathrm{k}_{\mathrm{U} 2}=3.82, \mathrm{k}_{\mathrm{L} 2}=4.45, \mathrm{k}_{\mathrm{U} 3}=4.49 \mathrm{k}_{\mathrm{L} 3}=4.22$. With these parameters the chart detects the shift $\mathbf{d}^{*}=(0.6,0.5,-0.2)^{\prime}$ ' with an $\mathrm{ARL}=13.34$. As shown by the program, the equivalent $\mathrm{T}^{2}$ control chart provides an out-of-control ARL equals to 19.18 , therefore the set of $\bar{X}$ charts is faster in detecting this shift.

The user may obtain plots to make a comparison of performance of both possible schemes (multiple $\bar{X}$ and $\mathrm{T}^{2}$ control chart) if s/he clicks on the tab "Evaluation vs $\mathrm{T}^{2}$ $(p=3)$ ". Figure 7 shows this comparison, with a very similar layout in comparison to the two variables interface. However, in the case of three variables, the user has to specify two of the three variables, in order to select the plane where the comparison will be made. If figure 7 is considered, it is possible to see, in the left hand side plot, a comparison in the plane $d_{1} d_{2}$ when $d_{3}=-0.2$. Point A shows where the shift $\mathrm{d}^{*}$ is located. As in the case of two variables, the plot shows that the set of $\bar{X}$ charts shows better performance when shifts close to Point A are considered. However, if symmetric performance is required, there is a version of the software that forces the control limits to be symmetric. A three dimensional plot is also available (like Figure 4), choosing two of the three variables used to draw the plot. This plot is not shown to reduce the length of the paper.

\section{[INSERT FIGURE 6 OVER HERE] [INSERT FIGURE 7 OVER HERE]}




\section{5.- Application to Charts based on Principal Components}

The procedure and programs described can be easily used for the optimization of charts based on principal components (PCs) as well. In this case, the value zero should be entered for the correlation coefficient(s) in the interface window (since the PCs are orthogonal), and the shift to be entered in this window should be the shift $\xi^{*}$ in the means of the PCs that is equivalent to the shift $\mathbf{d}^{*}$ in the means of the original variables, rather than the shift $\mathbf{d}^{*}$ itself. The transformed shift $\xi^{*}$ is expressed in units of standard deviation of the PCs, and can be easily determined from $\mathbf{d}^{*}$ in the following way.

Let the principal components be determined from the correlation matrix, $\rho$, rather than from the covariance matrix of the variables. This is recommended for statistical process control applications, since, if the variables have widely different variances, PCs based on the covariance matrix of the original non-standardized variables - and the corresponding control charts - may be insensitive to changes in the variables with less dispersion. Denote by $\lambda_{i}$ the $i$-th eigenvalue of $\rho, \lambda_{1}>\lambda_{2}>\ldots>\lambda_{p}$, and by $e_{i j}$ the $j$-th element of the corresponding $i$-th eigenvector of $\rho, j=1,2, \ldots p$.

The $i$-th principal component $Y_{i}, i=1,2, \ldots m, m \leq p$, is given by

$$
Y_{i}=\sum_{j=1}^{p} a_{i j} Z_{j}
$$

where $Z_{j}=\left(X_{j}-\mu_{0 j}\right) / \sigma_{0}, j=1,2, \ldots p$, and $a_{i j}=e_{i j}$. When the process is in control, $Z_{j} \sim$ $\mathrm{N}(0,1)$ and $Y_{i} \sim \mathrm{N}\left(0, \lambda_{i}\right)$. For control charts, however, the user has still the alternative (Jackson, 1985) of rescaling the PCs to unit variance, which is accomplished by making $a_{i j}=e_{i j} / \sqrt{\lambda_{i}}$. In this case, when the process is in control, $Y_{i} \sim \mathrm{N}(0,1), \forall i$.

Then, given the shift the user wants to detect quickly $\mathbf{d}^{*}=\left(d_{1}, d_{2}, \ldots, d_{p}\right)^{\prime}$ on the mean vector of the standardized original variables $Z_{j}, j=1,2, \ldots p$, the corresponding shift in the $i$-th PC (in units of standard deviations of this PC) is given by:

$$
\xi_{i}=\frac{1}{c_{i}} \sum_{j=1}^{p} a_{i j} d_{j}
$$


where $c_{i}$ denotes generically the in-control standard deviation of the $i$-th PC, that is, $c_{i}=1$ if the user is working with PCs rescaled to have unit variance and $c_{i}=\sqrt{\lambda_{i}}$ otherwise.

In matrix notation,

$$
\xi^{*}=\mathbf{C}^{-1} \mathbf{A d}^{*}
$$

where $\mathbf{d}^{*}$ is as defined before, $\mathbf{C}$ is a diagonal matrix with the PCs' standard deviations, and A is the $m \times p$ matrix of the PCs' coefficients $a_{i j}$.

The control limits returned by the program are in units of standard deviation, that is, the actual control limits for the chart on the $i$-th PC should be $U C L_{i}=k_{U i} c_{i} / \sqrt{n}$ and $L C L_{i}=-k_{L i} c_{i} / \sqrt{n}$ (note that with PC charts the sample sizes correspond to the number of measures of each original variable and must be equal, because each PC is a linear combination of all these variables). The control limit and ARL returned by the program for the $\mathrm{T}^{2}$ chart refer to the $\mathrm{T}^{2}$ chart of the principal components, not to the $\mathrm{T}^{2}$ chart of the original variables. In this way, the user can compare the performances of the set of optimized individual charts on each selected PC and of a single $\mathrm{T}^{2}$ chart on all such PCs. She or he may also run the program for the multiple $\bar{X}$ charts on the original variables as well, in order to obtain the results for these and for the $\mathrm{T}^{2}$ chart on the original variables, to compare the performances of the four control procedures before making a final decision.

Now, let us consider solving an example of an application. Suppose we want to monitor a process with 5 quality variables using only 2 of their PCs (obtained from the correlation matrix calculated with sufficient in-control historical data). The matrix of coefficients $a_{i j}$ is:

$$
\mathbf{A}=\left[\begin{array}{ccccc}
0.310610 & 0.478799 & 0.420526 & 0.455128 & 0.538785 \\
-0.64830 & -0.276183 & 0.653123 & -0.147705 & 0.234256
\end{array}\right]
$$

In this matrix, each row contains the coefficients of one of the retained PCs on the original variables. The variance of the first and second components are 2.898330 and 0.937889 , respectively. Suppose the shift on the original (standardized) variables 
against which maximum protection is desired is $\mathbf{d}^{*}=(0.5,-0.8,-0.7,0.8,-0.5)^{\prime}$. This will then correspond to a shift vector

$$
\xi *=\left[\begin{array}{cc}
\frac{1}{\sqrt{2.898330}} & 0 \\
0 & \frac{1}{\sqrt{0.937889}}
\end{array}\right] \mathbf{A} \mathbf{d}^{*}=(-0.2510458,-0.8216732)^{\prime}
$$

on the PCs.

If the user works with the PCs rescaled to unit variance, the shift vector $\xi^{*}$ would have the same value, since in this case $\xi *=\left[\begin{array}{ll}1 & 0 \\ 0 & 1\end{array}\right] \mathbf{A} \mathbf{d}^{*}$ but all elements of the first row of A will be divided by $\frac{1}{\sqrt{2.898330}}$ and all elements of the second row of A will be divided by $\frac{1}{\sqrt{0.937889}}$ relatively to the elements in the case that the PCs are not rescaled. This was expected, since changes in scale should not affect the relative shift (which is scaled in the same proportion) or the power of the charts.

The user then selects the "number of variables" $p=2$ (since "variables" in this case refer to the PC variables, and the " $p$ " in the interface window will in this case, as a matter of fact, refer to $m$, the number of PC variables considered), enters the values 0.0 for the correlation coefficient $r_{12}$, and -0.2510458 and -0.8216732 for $d_{1}$ and $d_{2}$ respectively, indicates the maximum sample size (which, for the purpose of this example, was set at 5) and checks the box "equal sample sizes". The user should also indicate the desired in-control ARL, which, for this example, is 200.

The optimum values found for the control limits are $k_{U 1}=4.21, k_{L 1}=-3.97$, $k_{U 2}=4.29$ and $k_{L 2}=-2.58$. Then the actual limits for the charts are $U C L_{1}=$ $\sqrt{2.898330 / 5} \times 4.21, L C L_{1}=\sqrt{2.898330 / 5} \times(-3.97), U C L_{2}=\sqrt{0.937889 / 5} \times 4.29$ and $L C L_{2}=\sqrt{0.937889 / 5} \times(-2.58)$. (If the user works with rescaled PCs, then the limits should be simply $U C L_{1}=(1 / \sqrt{5}) \times 4.21, L C L_{1}=(1 / \sqrt{5}) \times(-3.97), U C L_{2}=$ $(1 / \sqrt{5}) \times 4.29$ and $L C L_{2}=(1 / \sqrt{5}) \times(-2.58)$. The ARL for the specified shift is 4.365 with the pair of charts on the PCs and is 7.751 with the $\mathrm{T}^{2}$ chart on the PCs. 


\section{6.- Conclusions}

It is not easy for the practitioner who wishes to control a multivariate process to decide whether to employ multiple $\bar{X}$ charts or a single $\mathrm{T}^{2}$ control chart. No general decision rules exist, and the best choice depends upon many characteristics of each particular process, such as the correlations between the variables and the most common type of shifts.

With the aim of facilitating users in making correct decisions, we have developed a software tool that finds the optimal parameters of a set of $\bar{X}$ control charts (for the cases of two and three variables) employing Genetic Algorithms. This software also produces plots to compare the ARL performance of the solution with the performance of the corresponding $\mathrm{T}^{2}$ control chart. With a quite straightforward transformation in the input data, the software tool can also be employed to optimize a set of control charts based on Principal Components.

An interesting feature of the software tool is that it has two versions, one of them considering symmetric control limits for each chart, as usual, and the other admitting limits not necessarily symmetrical. The latter achieves far better solutions in some cases where shifts symmetric to the most relevant shift to detect are not important, such as may occur with processes with one-sided tolerances or when it is known that such shifts are simply not typical, to mention just two possibilities. The software tool, which runs in Windows ${ }^{\circledR}$ and is available from the authors, is a powerful tool for obtaining and comparing the optimal performances of different multivariate process control schemes and therefore facilitating the decision making process.

\section{Acknowledgments}

This work has been supported by the Ministry of Education and Science of Spain, research project number DPI2006-06124, including European FEDER funding, and the support of the ITESM-Foundation Carolina agreement, and also by CNPq (the Brazilian Council for Scientific and Technological Development), project number 201811/2007-3. 


\section{REFERENCES}

Alt, F. B. (1984) Multivariate Quality Control. In The Encyclopedia of Statistical Sciences, Kotz S., Johnson N. L. and Read C. R. (eds). John Wiley, New York, pp. 110-122.

Aparisi, F., Avendaño, G. and Sanz, J. (2006). Interpreting T² Control Chart Signals: Effectiveness of MTY Decomposition vs. a Neural Network. IIE Transactions, 38(8), 647-658.

Aparisi, F. and De Luna, M. (2008). The Design and Performance of the Multivariate Synthetic $\mathrm{T}^{2}$ Control Chart. Communications in Statistics: Theory and Methods (in press).

Aparisi, F. and García-Díaz, J. C. (2004). Optimization of Univariate and Multivariate Exponentially Weighted Moving Average Control Charts using Genetic Algorithms.: Computers and Operations Research, 31 (9), 1437-1454

Aparisi, F. and García-Díaz, J. C. (2007). Design and Optimization of EWMA Control Charts for In-control, Indifferent, and Out-of-control Regions. Computers \& Operations Research, 34(7), 2096-2108.

Champ, C. W. \& Aparisi, F. (2008) Double sampling Hotelling's T² charts, Quality and Reliability Engineering International, 24 (2), 153-166.

Crosier, R. B. (1988) Multivariate generalizations of cumulative sum quality control schemes, Technometrics, 30, 291-303.

Doganaksoy, N., Faltin, F. W. and Tucker, W. T. (1991), Identification of Out of Control Quality Characteristics in a Multivariate Manufacturing Environment. Communications in Statistics - Theory and Methods 20, 2775-2790.

Dunn, O. J. (1958), Estimation of the Means of Dependent Variables. Annals of Mathematical Statistics 29, 1095-1111.

Hayter, A. J. and Tsui, K.-L. (1994), Identification and Quantification in Multivariate Quality Control Problems. Journal of Quality Technology, 26(3), 197-208. 
He, D. \& Grigoryan A. (2006) Joint statistical design of double sampling $\bar{X}$ and S charts, European Journal of Operational Research, 168(1), 122-142.

Hotelling, H. (1947), Multivariate Quality Control, Illustrated by the Air Testing of Sample Bombsights. Techniques of Statistical Analysis, 111-184. New York, McGraw-Hill.

Jackson, J. E. (1985). Multivariate Quality Control. Communications in Statistics Theory and Methods 14(11), 2657-2688.

Jackson, J. E. (1991). A User's Guide to Principal Components, Wiley, New York, NY.

Johnson, R. A. \& Wichern, D. W. (2007). Applied Multivariate Statistical Analysis, 6th ed. Pearson Prentice Hall, Upper Saddle River, NJ.

Kourti, T. and MacGregor, J.F. (1996) Multivariate SPC methods for process and product monitoring. Journal Quality Technology,28, 409-428.

Lowry, C. A. and Montgomery, D. C. (1995). A review of multivariate control charts. IIE Transactions, 27, 800-810.

Lowry, C. A., Woodall, W. H., Champ, C. W. \& Rigdon, S.E. (1992) A multivariate exponentially weighted moving average control chart, Technometrics, 34, 46-53.

Mason, R. L., Tracy, N.D. and Young, J.C. (1995) Decomposition of $\mathrm{T}^{2}$ multivariate control chart interpretation. Journal of Quality Technology,27(2), 99-108.

Mason, R.L., Tracy, N.D. and Young, J.C. (1997) A practical approach for interpreting multivariate $\mathrm{T}^{2}$ control chart signals. Journal of Quality Technology,29(4), 396-406.

Montgomery, D.C. (2001) Introduction to Statistical Quality Control. 5th edition John Wiley. 
Pignatiello, J. J. and Runger, G. C. (1990), Comparisons of Multivariate CUSUM Charts. Journal of Quality Technology, 22, 173-186.

Runger, G. C. \& Alt, F. B. (1996). Choosing Principal Components for Multivariate Statistical Process Control. Communications in Statistics - Theory and Methods 25, 909-922.

Runger, G.C., Alt, F.B., and Montgomery, D.C. (1996) Contributors to a multivariate statistical process control chart signal. Communications in Statistics. Theory Methods,25, 2203-2213.

Runger, G. C. \& Montgomery, D. C. (1997), Multivariate and Univariate Process Control: Geometry and Shift Directions. Quality and Reliability Engineering International, 13(3), 153-158.

Schall, S. \& Chandra, J. (1987). Multivariate Quality Control Using Principal Components. International Journal of Production Research, 25, 571-588.

Serel, D. A., Moskowitz, H. and Tang, J. (2000), Univariate $\bar{X}$ Control Charts for Individual Characteristics in a Multinormal Model. IIE Transactions, 32, 1115-1125.

Sidak, Z. (1967), Rectangular Confidence Regions for the Means of Multivariate Normal Distributions, Journal of the American Statistical Association, 62, 626-633.

Vavak, F.; Fogarty, T.C., 1996, “A comparative study of steady state and generational genetic algorithms/", Evolutionary Computing: AISB Workshop, Lecture Notes in Computer Science vol. 1143, Springer. 


\begin{tabular}{|c|c|c|}
\hline Factor & $\boldsymbol{p = 2}$ & $\boldsymbol{p = 3}$ \\
\hline Evaluations & 20000 & 35000 \\
Population Size & 200 & 400 \\
Crossover Probability & 0.60 & 0.90 \\
Mutation Probability & 0.10 & 0.03 \\
Tournament Size & 2 & 2 \\
\hline
\end{tabular}

Table 1. Optimum levels for the parameters of the Genetic Algorithm.

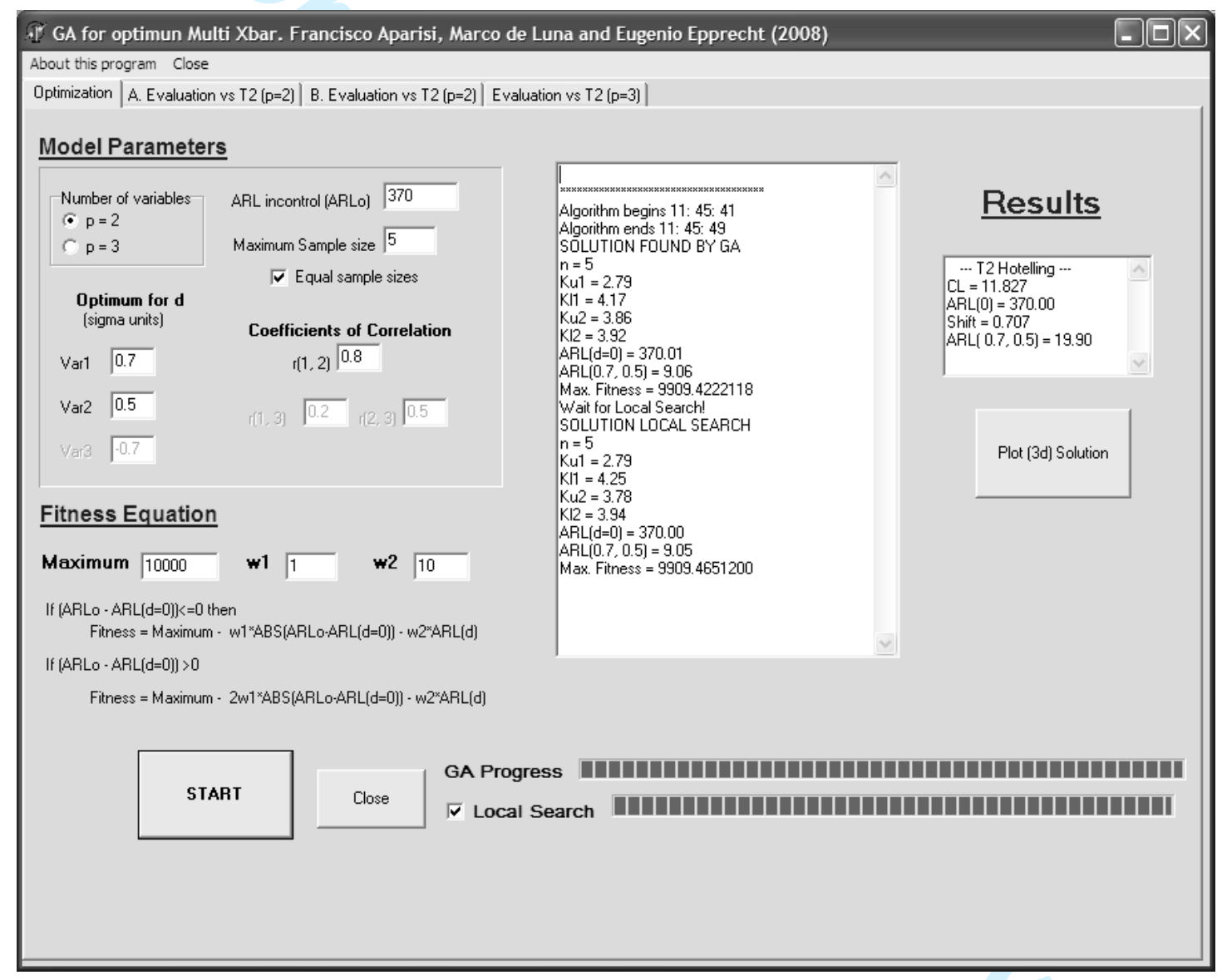

Figure 1. Software solving the example of application for $p=2$. 


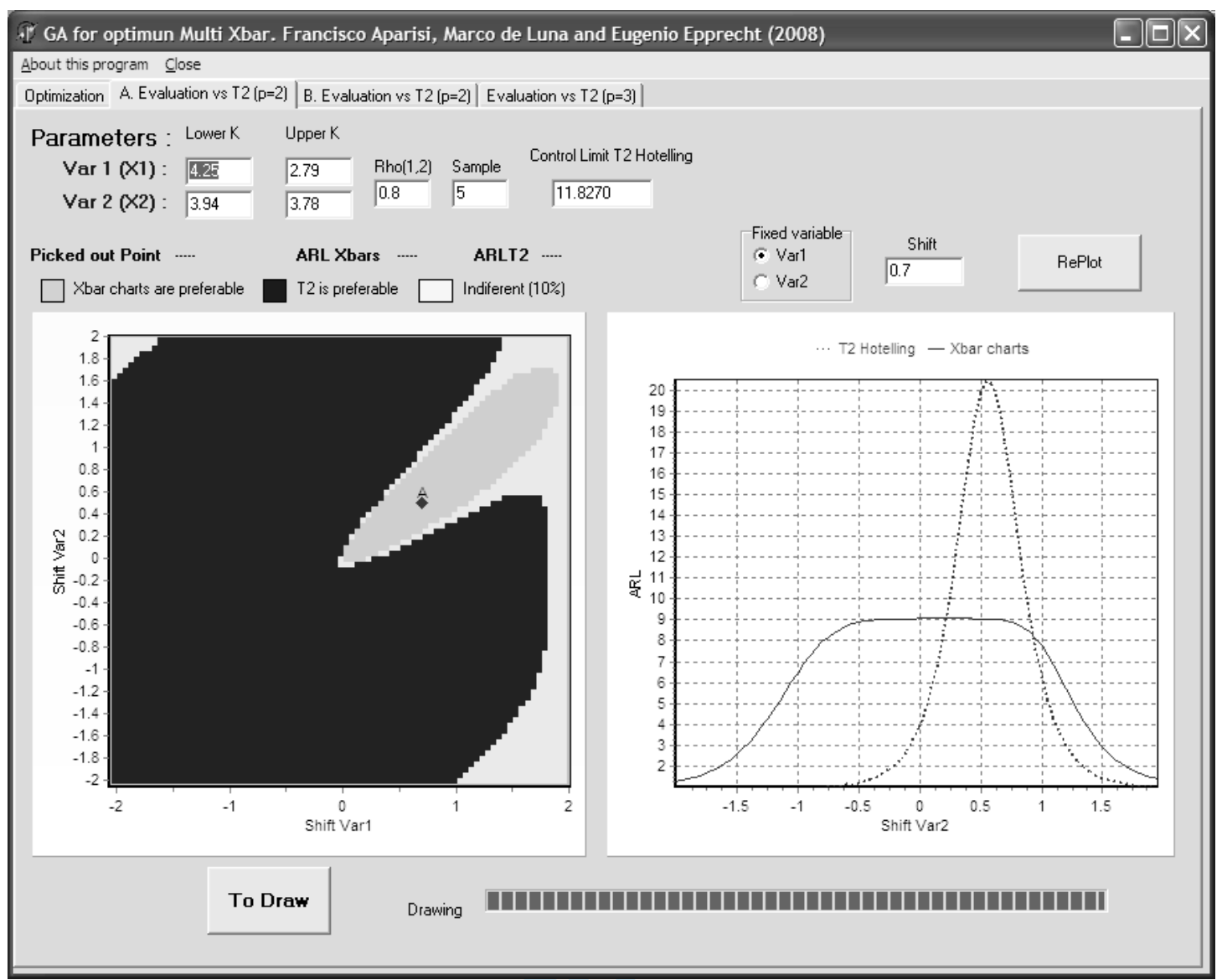

Figure 2. Comparison against the $\mathbf{T}^{2}$ control chart, asymmetric control limits.

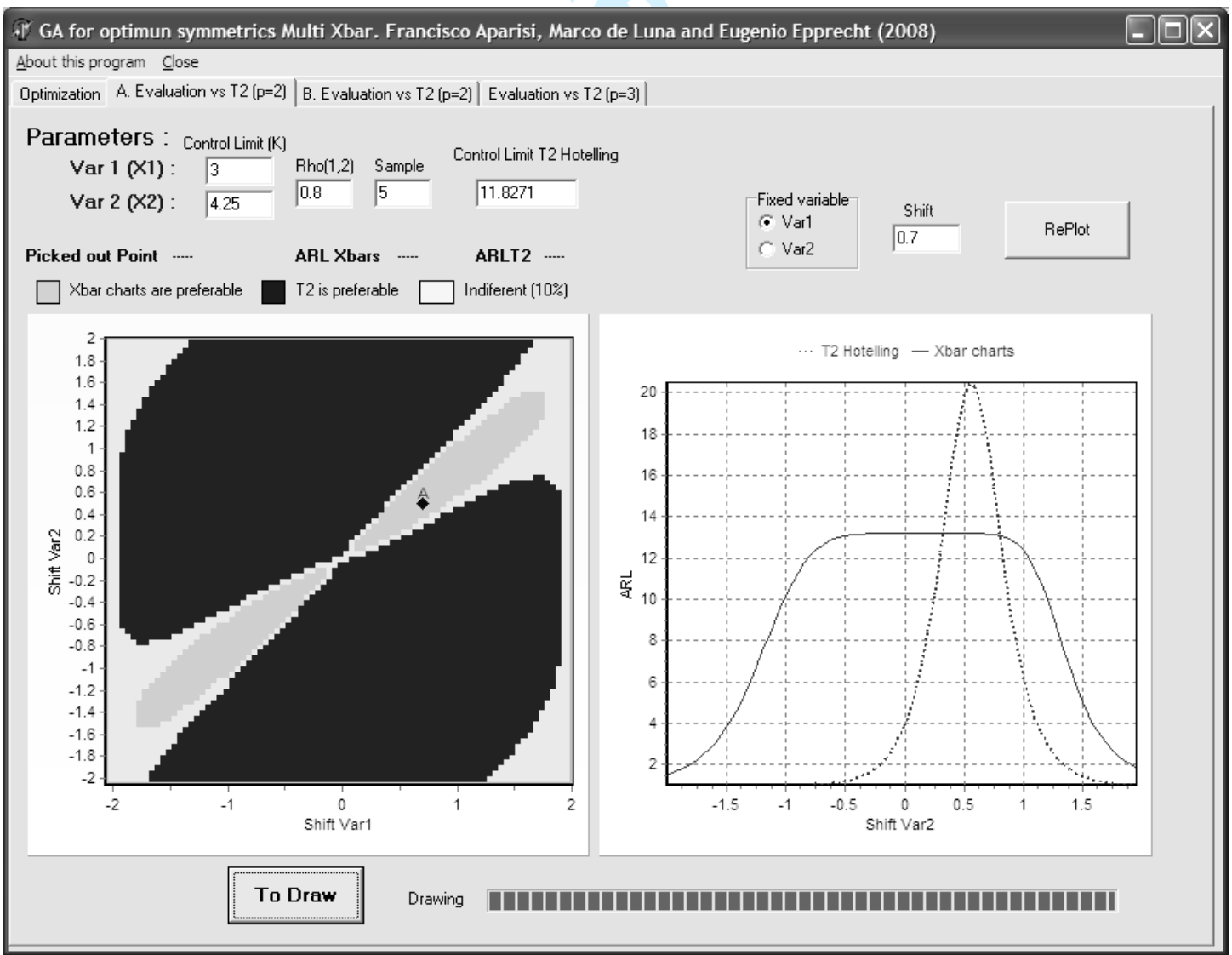

Figure 3. Comparison against the $T^{2}$ control chart, symmetric control limits. 


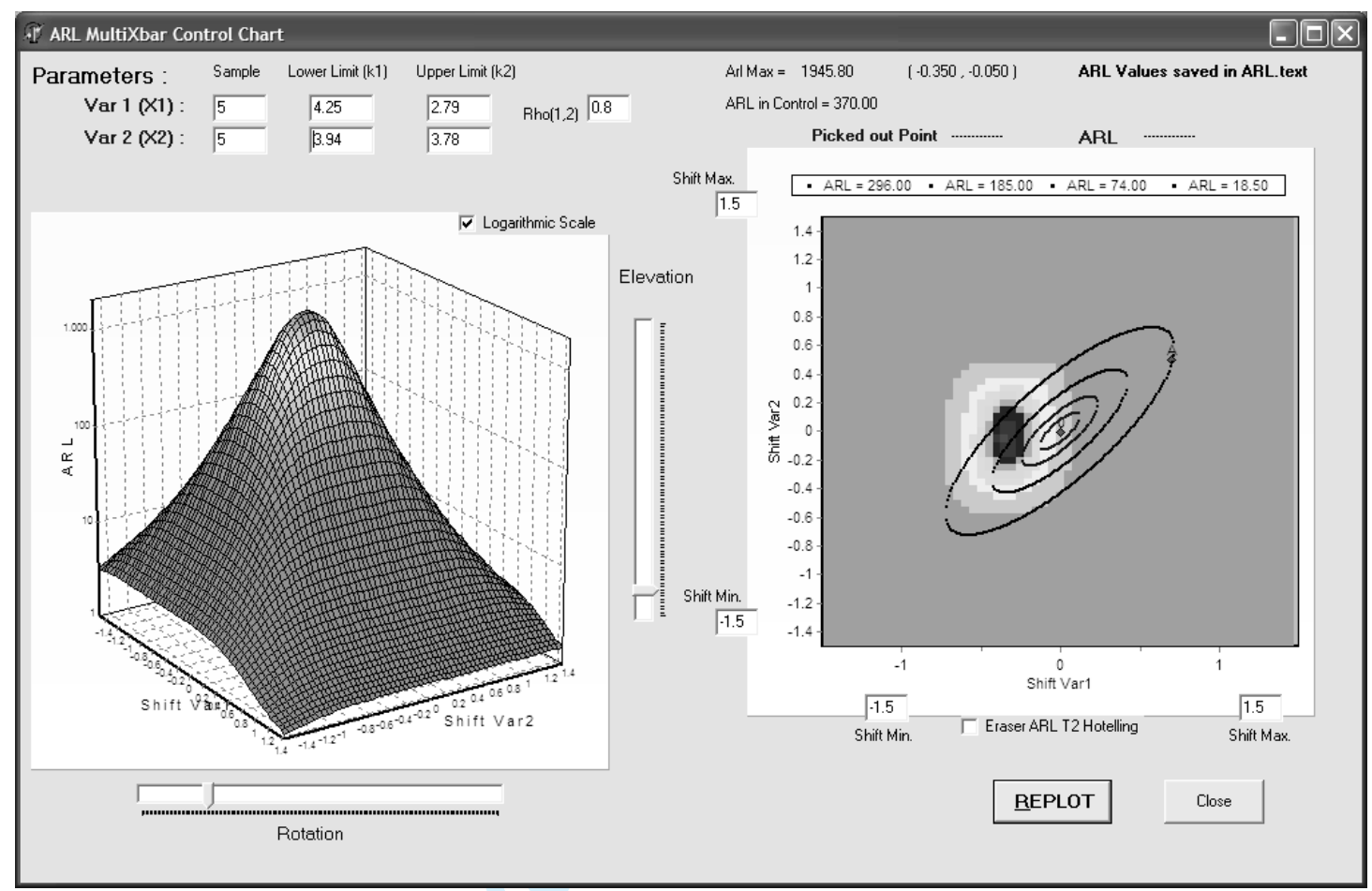

Figure 4. 3D and contour plots for comparison purposes, asymmetric case.

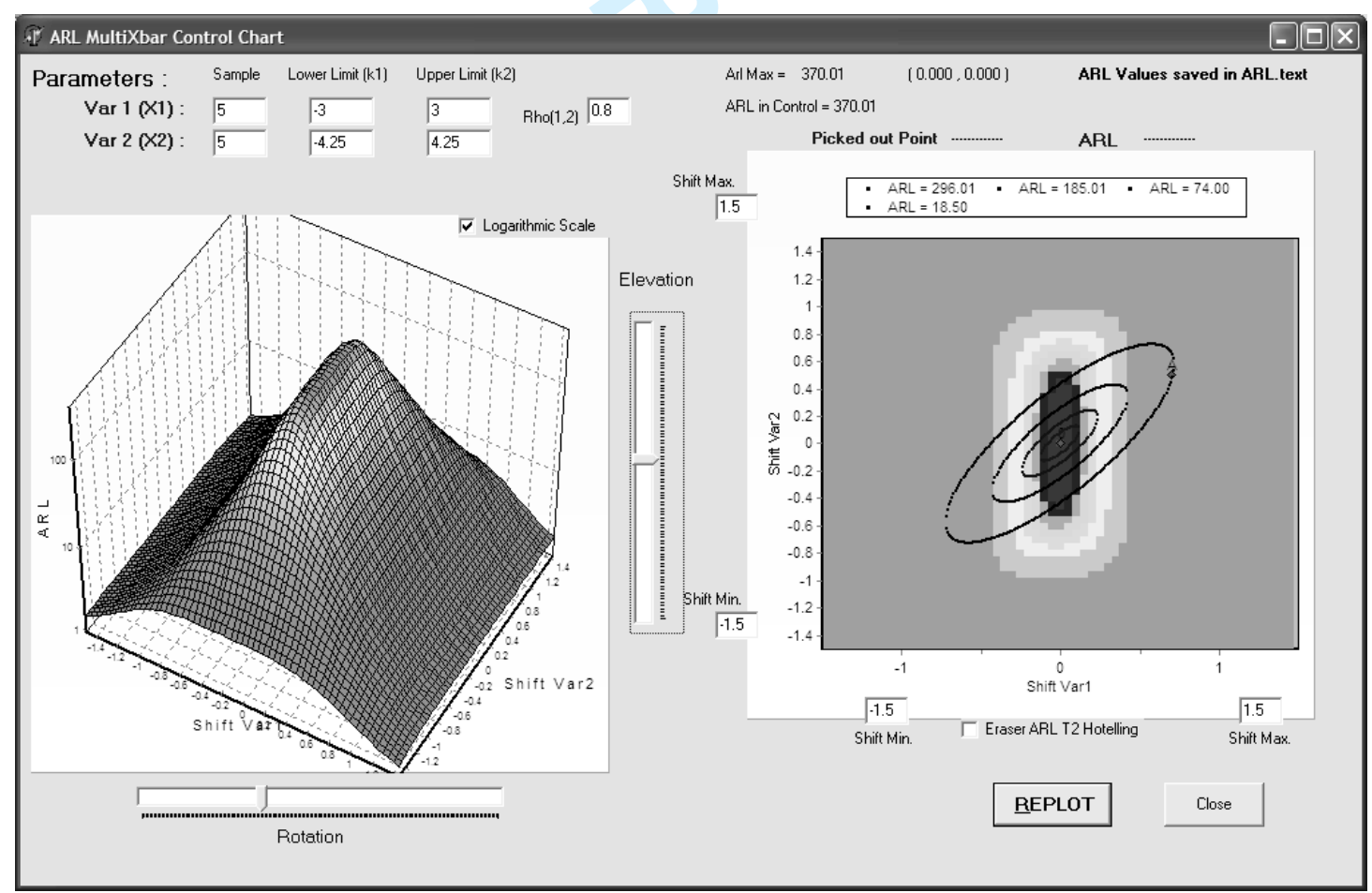

Figure 5. 3D and contour plots for comparison purposes, asymmetric case. 
Figure 6. Solution to the example of application, $p=3$.

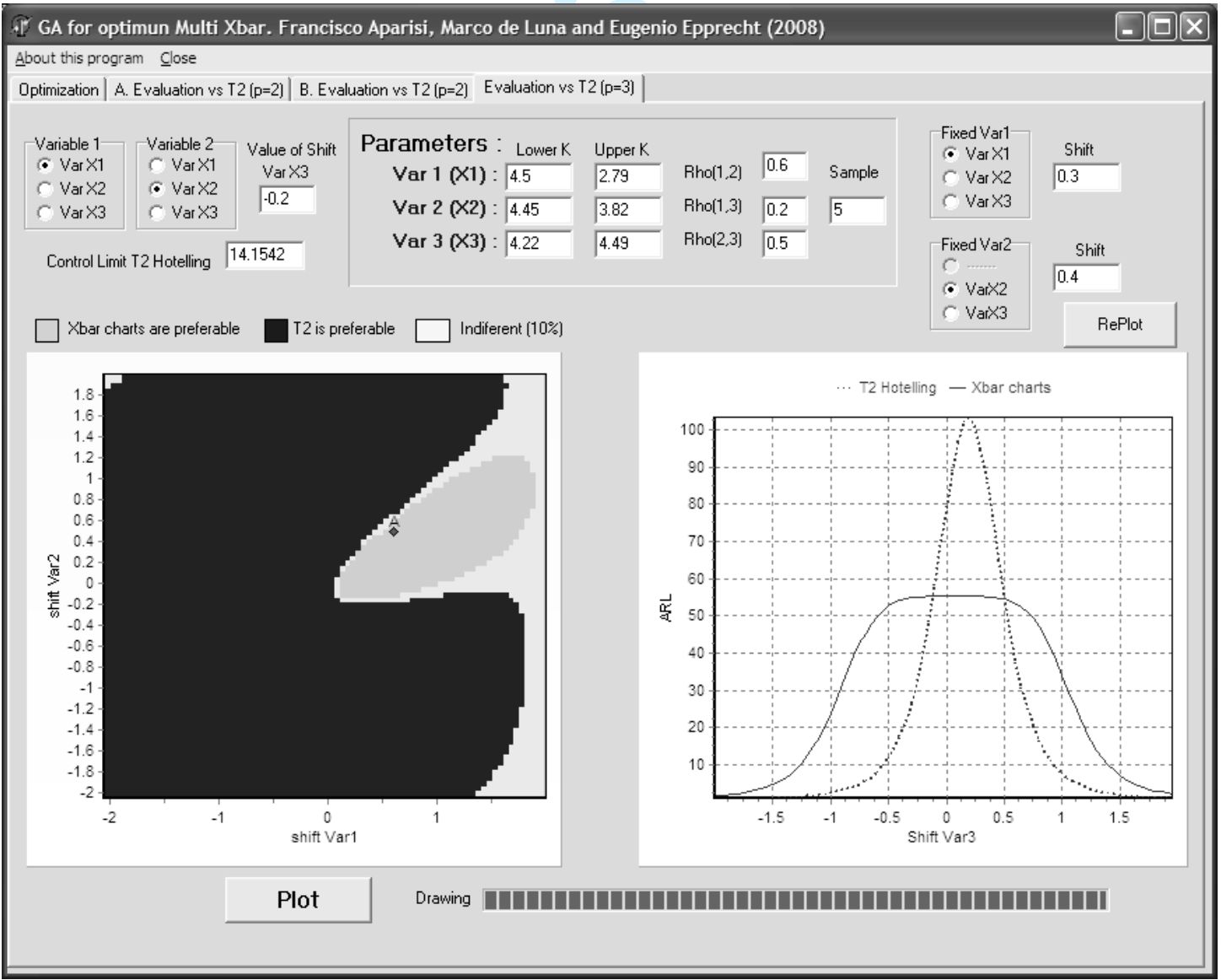

Figure 7. Comparison of performance for the example of application for $p=3$.

Optimization $\mid$ A. Evaluation vs $T 2(p=2) \mid$ B. Evaluation vs $T 2(p=2) \mid$ Evaluation vs $T 2(p=3) \mid$

\section{Model Parameters}

Number of variables

$C p=2$

$p=3$

Optimum for $d$ [sigma units]

Var1 $\longdiv { 0 . 6 }$

Var2 $\longdiv { 0 . 5 }$

Var3 $\longdiv { - 0 . 2 }$

\section{Fitness Equation}

Maximum $\sqrt { 1 0 0 0 0 } \quad \boldsymbol { w 1 } \longdiv { 1 } \quad \boldsymbol { w 2 } \longdiv { 1 0 }$

If $(A R L O-A R L(d=0))<=0$ then

Fitness $=$ Maximum $\cdot w 1 \times A B S(A R L O-A R L(d=0)) \cdot w 2 \times A R L(d)$

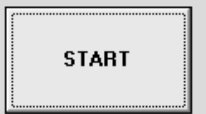

Close

$\checkmark$ Local Search

Fitness $=$ Maximum $-2 w 1 \times A B S(A R L O-A R L[d=0)] \cdot w 2^{*} A R L(d)$
Ku1 $=279$

$K 11=4.50$

$\mathrm{K} 12=4.45$

$K \mathrm{~K}=4.49$

$A R L[d=0]=370.01$

AR $(0,6,0.5,0.2)=13.34$

Max. Fitness $=9866.6103984$

\section{Results}

\section{-..- T2 Hotelling
$C L=14.154$}

$A R L(0)=370.01$

Shift $=0.782$

$\operatorname{RL}(0.6,0.5,-0.2]=19.18$ Plot (3d) Solution (1) $11=4.46$
$12=3.84$ $\mathrm{KI} 2=4.43$ $A R(0)=370.02$

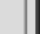

